\title{
Correction to: BV and BFV for the H-Twisted Poisson Sigma Model
}

\author{
Noriaki Ikeda and Thomas Stroble
}

\section{Correction to: Ann. Henri Poincaré https://doi.org/10.1007/s00023-020-00988-0}

Equation (5.20),

$$
\begin{aligned}
S_{B V}= & \int_{T[1] \Sigma} d^{2} \sigma d^{2} \theta\left[\boldsymbol{A}_{i} \mathbf{d} x^{i}+\frac{1}{2} \pi^{i j}(x) \boldsymbol{A}_{i} \boldsymbol{A}_{j}\right]+\int_{T[1] N} d^{3} \sigma d^{3} \theta H(x) \\
& +\int_{T[1] \Sigma} d^{2} \sigma d^{2} \theta\left[\frac{1}{4}\left(\pi^{i l} \pi^{j m} H_{l m k}\right)(x) \boldsymbol{A}_{i} \boldsymbol{A}_{j} \varepsilon x^{k}-\frac{1}{2}\left(\pi^{i l} H_{j k l}\right)(x) \boldsymbol{A}_{i}\left(\mathbf{d} x^{j}\right) \varepsilon x^{k}\right] \\
& +\int_{T[1] \Sigma} d^{2} \sigma d^{2} \theta\left[\frac{1}{8}\left(\pi^{i m} \pi^{j n} \pi^{p q} H_{m q l} H_{n p k}\right)(x) \boldsymbol{A}_{i} \boldsymbol{A}_{j}\left(\varepsilon x^{k}\right) \varepsilon x^{l}\right],
\end{aligned}
$$

should read as follows:

$$
\begin{aligned}
S_{B V}= & \int_{T[1] \Sigma} d^{2} \sigma d^{2} \theta\left[\boldsymbol{A}_{i} \mathbf{d} x^{i}+\frac{1}{2} \pi^{i j}(x) \boldsymbol{A}_{i} \boldsymbol{A}_{j}\right]+\int_{T[1] N} d^{3} \sigma d^{3} \theta H(x) \\
& +\int_{T[1] \Sigma} d^{2} \sigma d^{2} \theta\left[\frac{1}{4}\left(\pi^{i l} \pi^{j m} H_{l m k}\right)(x) \boldsymbol{A}_{i} \boldsymbol{A}_{j} \varepsilon x^{k}+\frac{1}{2}\left(\pi^{i l} H_{j k l}\right)(x) \boldsymbol{A}_{i}\left(\mathbf{d} x^{j}\right) \varepsilon x^{k}\right] \\
& +\int_{T[1] \Sigma} d^{2} \sigma d^{2} \theta\left[\frac{1}{8}\left(\pi^{i m} \pi^{j n} \pi^{p q} H_{m q l} H_{n p k}\right)(x) \boldsymbol{A}_{i} \boldsymbol{A}_{j}\left(\varepsilon x^{k}\right) \varepsilon x^{l}\right] .
\end{aligned}
$$

Publisher's Note Springer Nature remains neutral with regard to jurisdictional claims in published maps and institutional affiliations.

The original article can be found online at https://doi.org/10.1007/s00023-020-00988-0. 
Noriaki Ikeda

Department of Mathematical Sciences

Ritsumeikan University

Kusatsu Shiga 525-8577

Japan

e-mail: nikeda@se.ritsumei.ac.jp

Thomas Strobl

Institut Camille Jordan

Université Claude Bernard Lyon 1

43 Boulevard du 11 Novembre 1918

69622 Villeurbanne Cedex

France

e-mail: strobl@math.univ-lyon1.fr 